\title{
Fluctuations and response in financial markets: the subtle nature of 'random' price changes
}

\author{
Jean-Philippe Bouchaud ${ }^{\dagger, *}$, Yuval Gefen ${ }^{\times}$, \\ Marc Potters*, Matthieu Wyart $^{\dagger}$
}

February 2, 2008

\author{
$\dagger$ Commissariat à l'Energie Atomique, Orme des Merisiers \\ 91191 Gif-sur-Yvette CEDEX, France \\ * Science \& Finance, Capital Fund Management, 109-111 rue Victor Hugo \\ 92532 Levallois CEDEx, France \\ × Condensed Matter Physics Department, Weizmann Institute of Science, \\ 76100 Rehovot, Israel \\ February 2, 2008
}

\begin{abstract}
Using Trades and Quotes data from the Paris stock market, we show that the random walk nature of traded prices results from a very delicate interplay between two opposite tendencies: long-range correlated market orders that lead to super-diffusion (or persistence), and mean reverting limit orders that lead to sub-diffusion (or anti-persistence). We define and study a model where the price, at any instant, is the result of the impact of all past trades, mediated by a non constant 'propagator' in time that describes the response of the market to a single trade. Within this model, the market is shown to be, in a precise sense, at a critical point, where the price is purely diffusive and the average response function almost constant. We find empirically, and discuss theoretically, a fluctuationresponse relation. We also discuss the fraction of truly informed market orders, that correctly anticipate short term moves, and find that it is quite small.
\end{abstract}




\section{Introduction}

The Efficient Market Hypothesis (EMH) posits that all available information is included in prices, which emerge at all times from the consensus between fully rational agents, that would otherwise immediately arbitrage away any deviation from the fair price [1, 2]. Price changes can then only be the result of unanticipated news and are by definition totally unpredictable. The price is at any instant of time the best predictor of future prices. One of the central predictions of EMH is thus that prices should be random walks in time which (to a good approximation) they indeed are. This was interpreted early on as a success of EMH. However, as pointed out by Schiller, the observed volatility of markets is far too high to be compatible with the idea of fully rational pricing [3]. The frantic activity observed in financial markets is another problem: on liquid stocks, there is typically one trade every 5 seconds, whereas the time lag between of relevant news is certainly much larger. More fundamentally, the assumption of rational, perfectly informed agents seems intuitively much too strong, and has been criticized by many [4, 5, 6]. Even the very concept of the fair price of a company appears to be somewhat dubious.

There is a model at the other extreme of the spectrum where prices also follow a pure random walk, but for a totally different reason. Assume that agents, instead of being fully rational, have zero intelligence and take random decisions to buy or to sell, but that their action is interpreted by all the others agents as potentially containing some information. Then, the mere fact of buying (or selling) typically leads to a change of the ask $a(t)$ (or bid $b(t)$ ) price and hence of a change of the midpoint $m(t)=[a(t)+b(t)] / 2$. In the absence of reliable information about the 'true' price, the new midpoint is immediately adopted by all other market participants as the new reference price around which new orders are launched. In this case, the midpoint will also follow a random walk (at least for sufficiently large times), even if trades are not motivated by any rational decision and devoid of meaningful information. ${ }^{1}$ This alternative, random trading model has been recently the object of intense scrutiny, in particular as a simplified approach to the statistics of order books [8, 9, 10, 11, 12, 13, 14, 15. Since the order flow is a Poisson process, this assumption is quite convenient and leads to tractable analytical models [14, 16]. Perhaps surprisingly, many qualitative (and sometimes quantitative) properties of order books can be predicted using such an extreme postulate [12, 13, 14, 17].

Of course, reality should lie somewhere in the middle: clearly, the price cannot wander arbitrarily far from a reasonable value, and trades cannot all be random. The interesting question is to know which of the two pictures is closest to reality and can be taken as a faithful starting point around which improvements can be

\footnotetext{
${ }^{1}$ That this simplistic model also leads to a random walk behaviour for prices has also very recently been pointed out in [7].
} 
perturbatively added.

In this paper, we want to argue, based on a series of detailed empirical results obtained on trade by trade data, that the random walk nature of prices is in fact highly non trivial and results from a fine-tuned competition between two populations of traders, liquidity providers ('market-makers') on the one hand, and liquidity takers (sometimes called 'informed traders', but see the discussion in Section 4). For reasons that we explain in more details below, liquidity providers act such as to create anti-persistence (or mean reversion) in price changes that would lead to a sub-diffusive behaviour of the price, whereas liquidity takers' action leads to long range persistence and super-diffusive behaviour. Both effects very precisely compensate and lead to an overall diffusive behaviour, at least to a first approximation, such that (statistical) arbitrage opportunities are absent, as expected. However, one can spot out the vestiges of this subtle compensation from the temporal structure of the market impact function (which measures how a given trade affects on average future prices).

The organization of this paper is as follows. We first present (Section 2) our empirical results on the statistics of trades, market impact and fluctuations. We show in particular that the order flow exhibits long range autocorrelations in time, but that this does not lead to any predictability in price changes, as also recently noticed in [7]. Then, we introduce in Section 3 a simple model that expresses the price as a linear superposition of the impact of each trade. We show that this model allows to rationalize our empirical findings, provided a specific relation between the temporal autocorrelation of the sign of the trades (i.e. buyer initiated or seller initiated) and the temporal response to a single trade is satisfied. Finally, in Section 4, we give intuitive arguments that allow one to understand the market forces at the origin of this subtle balance between two opposite effects, which dynamically leads to absence of statistical arbitrage opportunities. We argue that in a very precise sense, the market is sitting on a critical point; the dynamical compensation of two conflicting tendencies is similar to other complex systems such as the heart [18, driven by two antagonist systems (sympathetic and para-sympathetic), or certain human tasks, such as balancing of a long stick [19]. The latter example illustrates very clearly the idea of dynamical equilibrium, and shows how any small deviation from perfect balance may lead to strong instabilities. This near instability may well be at the origin of the fat tails and volatility clustering observed in financial data (see e.g. [20, 21, 22, 23, 24, 25]). Note that these two features are indeed present in the 'balancing stick' time series studied in 19 . 


\section{Market impact and fluctuations}

\subsection{Presentation of the data and definitions}

In this study, we have analyzed trades and quotes data from liquid French stocks in the years 2001 and 2002, although qualitatively similar results were also obtained on British stocks as well. The advantage of the French market, however, is that it is fully electronic whereas only part of the volume is traded electronically in the London stock exchange. We will illustrate our results mainly using the France-Telecom stock, which is one of the most actively traded stocks, for which statistics are particularly good.

There are two data files for each stock: one gives the list of all successive quotes, i.e. the best buy (bid, b) and sell (ask, a) prices, together with the available volume, and the time stamp accurate to the second. A quote can change either as a result of a trade, or because new limit orders appear, or else because some limit orders are canceled. The other data file is the list of all successive trades, with the traded price, traded volume and time stamp, again accurate to the second. Sometimes, several trades are recorded at the very same instant but at different prices: this corresponds to a market order of a size which exceeds the available volume at the bid (or at the ask), and hits limit orders deeper in the order book. In the following, we have grouped all these trades together as a single trade. This allows one to create chronological sequences of trades and quotes, such that between any two trades there is at least one quote.

The last quote before a given trade allows one to define the sign of each trade: if the traded price is above the last midpoint $m=(a+b) / 2$, this means that the trade was triggered by a market order (or marketable limit order) to buy, and we will assign to that trade a variable $\varepsilon=+1$. If, one the other hand the traded price is below the last midpoint $m=(a+b) / 2$, then $\varepsilon=-1$. With each trade is also associated a volume $V$, corresponding to the total number of shares exchanged.

Trades appear at random times, the statistics of which being itself non trivial (there are intra-day seasonalities and also clustering of the trades in time). We will not be interested in this aspect of the problem and always reason in terms of trade time, i.e. time advances by one unit every time a new trade (or a series of simultaneous trades) is recorded. We have also systematically discarded the first ten and the last ten minutes of trading in a given day, to remove any artifacts due to the opening and closing of the market. Many quantities of interest in the following are two-time observables, that is, compare two observables at (trade) time $n$ and $n+\ell$. In order to avoid overnight effects, we have restricted our analysis to intra-day data, i.e. both $n$ and $n+\ell$ belong to the same trading day. We have also assumed that our observables only depend on the time lag $\ell$.

On the example of France-Telecom, on which we will focus mostly, there are on the order of 10000 trades per day. For example, the total number of trades on France-Telecom during 2002 was close to $2.10^{6}$; this allows quite accurate 
statistical estimates of various quantities. The volume of each trade was found to be roughly $\log$-normally distributed, with $\langle\ln V\rangle \simeq 5.5$ and a root mean square of $\Delta \ln V \simeq 1.8$. The range of observed values of $\ln V$ is between 1 and 11 .

\subsection{Price fluctuation and diffusion}

The simplest quantity to study is the average mean square fluctuation of the price between (trade) time $n$ and $n+\ell$. Here, the price $p_{n}$ is defined as the mid-point before the $n$th trade: $p_{n} \equiv m_{n^{-}}$. In this paper, we always consider detrended prices, such that the empirical drift is zero. We thus define $\mathcal{D}(\ell)$ as:

$$
\mathcal{D}(\ell)=\left\langle\left(p_{n+\ell}-p_{n}\right)^{2}\right\rangle .
$$

As is well known, in the absence of any linear correlations between successive price changes, $\mathcal{D}(\ell)$ has a strictly diffusive behaviour, i.e.

$$
\mathcal{D}(\ell)=D \ell
$$

where $D$ is a constant. In the presence of short-ranged correlations, one expects deviations from this behaviour at short times. However, on liquid stocks with relatively small tick sizes such as France-Telecom (FT), one finds a remarkably linear behaviour for $\mathcal{D}(\ell)$, even for small $\ell$. The absence of linear correlations in price changes is equivalent to saying that (statistical) arbitrage opportunies are absent, even for high frequency trading. In fact, in order to emphasize the differences from a strictly diffusive behaviour, we have studied the quantity $\sqrt{\mathcal{D}(\ell) / \ell}$ (which has the dimension of Euros). We show this quantity in Fig. 1 for FT, averaged over three different periods: first semester of 2001 (where the tick size was 0.05 Euros), second semester of 2001, and the whole of 2002 (where the tick size was 0.01 Euros). One sees that $\mathcal{D}(\ell) / \ell$ is indeed nearly constant, with a small 'oscillation' on which we will comment later. Similar plots can be observed for other stocks (see Fig. 2). We have noted that for stocks with larger ticks, a slow decrease of $\mathcal{D}(\ell) / \ell$ is observed, corresponding to a slight anti-persistence (or sub-diffusion) effect.

The conclusion is that the random walk (diffusive) behaviour of stock prices appears even at the trade by trade level, with a diffusion constant $D$ which is of the order of the typical bid-ask squared. From Fig. 1, one indeed sees that

$\sqrt{\mathcal{D}(1)} \sim 0.01$ Euros, which is precisely the tick size, and FT has a typical bidask spread equal to one or two ticks. This coincidence is interesting. It might suggests that price changes are to a large extent induced by the trading activity itself, independently of real news (unless of course if the news flow is itself on the scale of seconds and that each news item has an impact on the price that is commensurate to the bid-ask spread). Much stronger arguments in favor of this point, based on estimates of the fraction of informed trades, will be given below. 


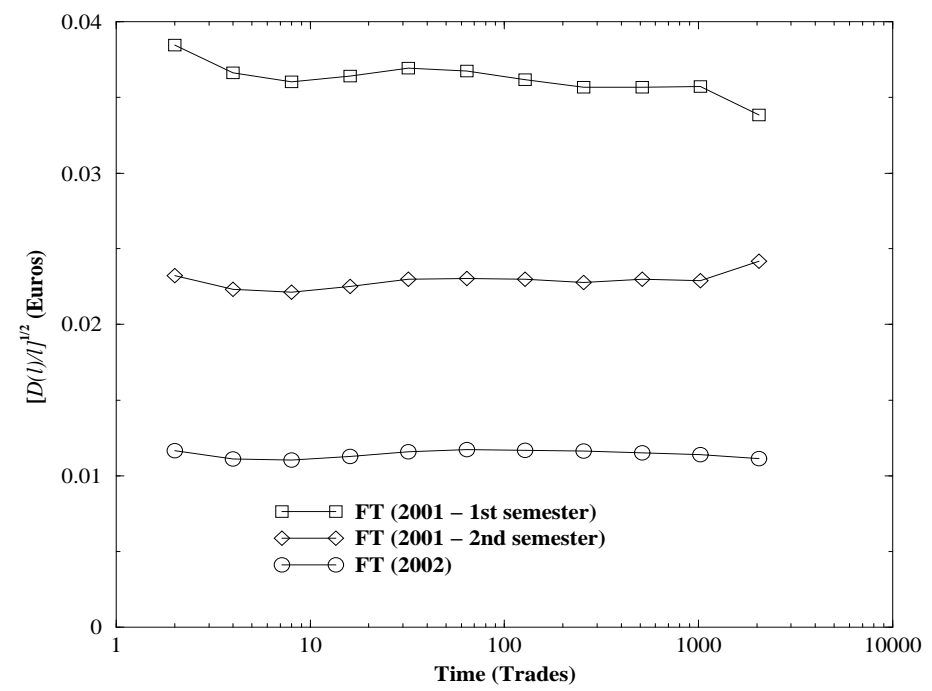

Figure 1: Plot of $\sqrt{\mathcal{D}(\ell) / \ell}$ as a function of $\ell$ for France-Telecom, during three different periods. The variation of $\mathcal{D}(\ell) / \ell$ with $\ell$ is very small, in particular in the small tick (0.01 Euros) period (July 2001 - December 2002). For the large tick size period (0.05 Euros; January 2001 - June 2001), there is a systematic downward trend: see also Fig. 2.

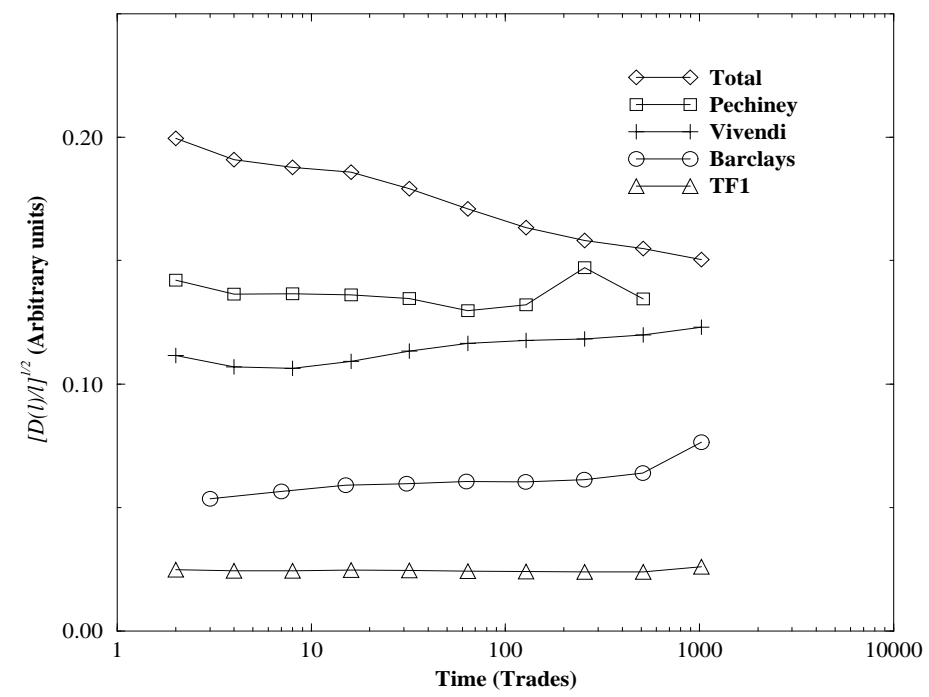

Figure 2: Plot of $\sqrt{\mathcal{D}(\ell) / \ell}$ as a function of $\ell$ for other stocks during the year 2002, except Barclays (May-June 2002). The $y$-axis has been rescaled arbitrarily for clarity. We note that stocks with larger tick size tend to reveal a stronger mean-reverting effect. 
This conclusion seems to imply that the price may, on the long run, wander arbitrarily far from the fundamental price, which would be absurd. However, even if one assumes that the fundamental price is independent of time, a typical $3 \%$ noise induced daily volatility would lead to a significant (say a factor 2) difference between the traded price and the fundamental price only after a few years [26]. Since the fundamental price of a company is probably difficult to determine better than within a factor two, say (see e.g. [5, 27]), one only expects fundamental effects to be relevant on very long time scales (as indeed suggested by the empirical results of de Bondt and Thaler [28]), but that these are totally negligible on short (intra-day) time scales of interest here. We will in fact see below (cf. Eq. (27) that the reference price that market participants seem to have in mind is in fact a short time average of the past price itself, rather than any fundamental price.

\subsection{Response function and market impact}

In order to better understand the impact of trading on price changes, one can study the following response function $\mathcal{R}(\ell)$, defined as:

$$
\mathcal{R}(\ell)=\left\langle\left(p_{n+\ell}-p_{n}\right) \cdot \varepsilon_{n}\right\rangle,
$$

where $\varepsilon_{n}$ is the sign of the $n$-th trade, introduced in Section 2.1. The quantity $\mathcal{R}(\ell)$ measures how much, on average, the price moves up conditioned to a buy order at time 0 (or a sell order moves the price down) a time $\ell$ later. As will be clear below,this quantity is however not the market response to a single trade, a quantity that will later be denoted by $G_{0}$. A more detailed object can in fact be defined by conditioning the average to a certain volume $V$ of the $n$-th trade:

$$
\mathcal{R}(\ell, V)=\left.\left\langle\left(p_{n+\ell}-p_{n}\right) \cdot \varepsilon_{n}\right\rangle\right|_{V_{n}=V} .
$$

Previous empirical studies have mostly focused on the volume dependence of $\mathcal{R}(\ell, V)$, and established that this function is strongly concave as a function of the volume [29, 30, 31, 32, 7]. In [33, a thorough analysis of U.S. stocks was analyzed in terms of a piecewise power-law dependence for $\mathcal{R}(\ell=1, V) \propto V^{\alpha}$, with an exponent $\alpha \simeq 0.4$ for small volumes, and a smaller value $(\alpha \simeq 0.2)$ for larger volumes. In a previous publication [34], some of us have proposed that this dependence might in fact be logarithmic (see also a footnote in [32]): $\mathcal{R}(\ell=1, V)=R_{1} \ln V$ (where $R_{1}$ is a stock dependent constant), a law that seems to satisfactorily account for all the data that we have analyzed. The empirical determination of the temporal structure of $\mathcal{R}(\ell, V)$ has been much less investigated (although one can find in [32] somewhat related results on a coarsegrained version of $\mathcal{R}(\ell, V)$ ). Preliminary empirical results, published in [34,

reported that $\mathcal{R}(\ell, V)$ could be written in a factorized form (first suggested on theoretical grounds in [12):

$$
\mathcal{R}(\ell, V) \approx \mathcal{R}(\ell) f(V) ; \quad f(V) \propto \ln V,
$$




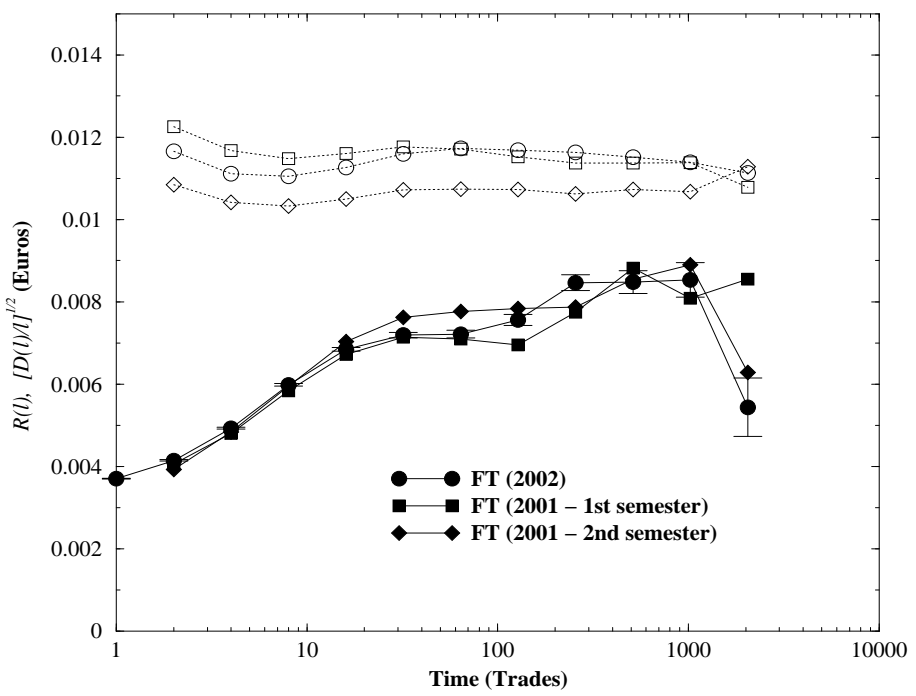

Figure 3: Average response function $\mathcal{R}(\ell)$ for $\mathrm{FT}$, during three different periods (black symbols). We have given error bars for the 2002 data. For the 2001 data, the $y$-axis has been rescaled to best collapse onto the 2002 data. Using the same rescaling factor, we have also shown the data of Fig. 1. The fact that the same rescaling works approximately for $\mathcal{D}(\ell)$ as well will be dwelled further in Section 2.4 below.

where $\mathcal{R}(\ell)$ is a slowly varying function that initially increases up to $\ell \sim 100-1000$ and then is seen to decrease back, with a rather small overall range of variation. The initial increase of $R(\ell)$ was reported in [29] and has also recently been noticed by Lillo and Farmer [17]. Here, we provide much better data that supports both the above assertions. We show for example in Fig. 3 the temporal structure of $\mathcal{R}(\ell)$ for France Telecom, for different periods. Note that $\mathcal{R}(\ell)$ increases by a factor $\sim 2$ between $\ell=1$ and $\ell=\ell^{*} \approx 1000$, before decreasing back. Similar results have been obtained for many different stocks as well: Fig. 4 shows a small selection of other stocks, where the non monotonous behaviour of $\mathcal{R}(\ell)$ is shown. However, in some cases (such as Pechiney), the maximum is not observed. One possible reason is that the number of daily trades is in this case much smaller $(\sim 1000)$, and that $\ell^{*}$ is beyond the maximum intra-day time lag.

The existence of a time scale $\ell^{*}$ beyond which $\mathcal{R}(\ell)$ decreases is thus both statistically significant, and to a large degree independent of the considered stock. On the other hand, the amplitude of the change of $\mathcal{R}(\ell)$ seems to be stock dependent. As will be clear later, the slowly varying nature of $\mathcal{R}(\ell)$ and the fact that this quantity reaches a maximum are non trivial results that will require a specific interpretation.

Turning now to the factorization property of $\mathcal{R}(\ell, V)$, Eq. (5), we illustrate its validity in Fig. 5, where $\mathcal{R}(\ell, V) / f(V)$ is plotted as a function of $\ell$ for different 


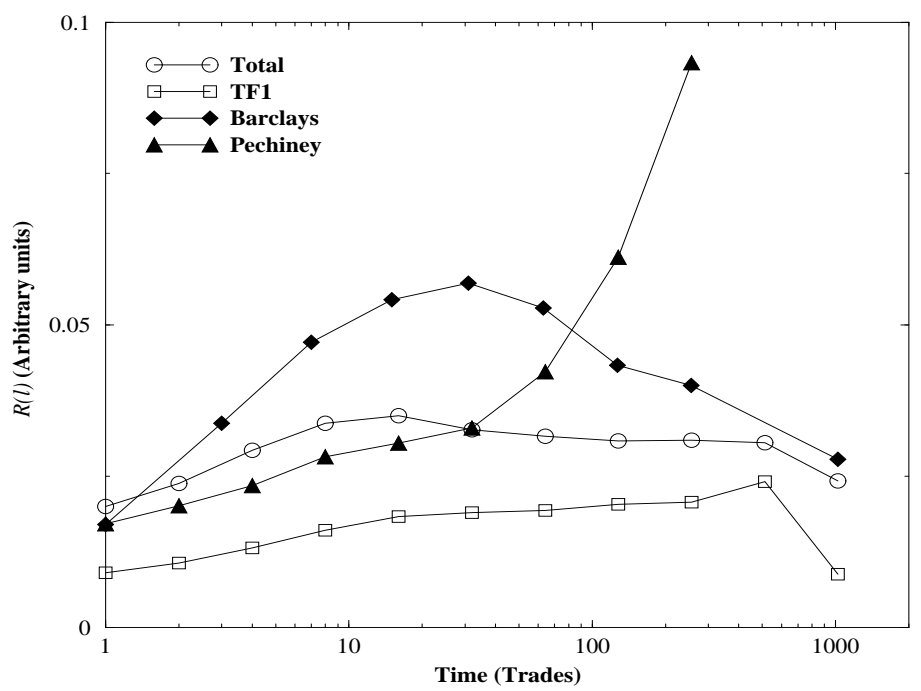

Figure 4: Average response function $\mathcal{R}(\ell)$ for a restricted selection of stocks, during the year 2002 .

values of $V$. The function $f(V)$ was chosen for best visual rescaling, and is found to be close to $f(V)=\ln V$, as expected. Note that for the smallest volume (open circles), the long time behaviour of $\mathcal{R}(\ell, V)$ seems to be different, which is probably due to the fact that small volumes are in fact more likely to be large volumes chopped up into small pieces.

One has to keep in mind that the response function $\mathcal{R}(\ell)$ captures a small systematic effect that relates the average price change to the sign of a trade. However, the fluctuations around this small signal are large, and increase with $\ell$. A way to see this is to introduce the random variable $u_{\ell}=\left(p_{n+\ell}-p_{n}\right) \cdot \varepsilon_{n}$. By definition, $\mathcal{R}(\ell)$ is the average of $u_{\ell}$, and $\mathcal{D}(\ell)$ is the average of $u_{\ell}^{2}$. Since $\mathcal{R}(\ell)$ is roughly constant whereas $\mathcal{D}(\ell)$ grows linearly with $\ell$, one sees that the impact of a given trade (as measured by $\mathcal{R}(\ell)$ ) rapidly becomes lost in the fluctuations.

In Fig. 6, we show the whole empirical distribution $P\left(u_{\ell}\right)$ of $u_{\ell}$ for $\ell=128$ (but other values of $\ell$ lead to similar results). This distribution is found to be only slightly skewed in the direction of positive $u_{\ell}$. In fact, if one considers the shifted variable $u_{\ell}-\nu$, where $\nu=0.01$ Euros, the distribution becomes nearly symmetric. Note that 0.01 Euros is equal to half the typical bid-ask spread and can therefore be seen as the cost of a market order. The Efficient Market picture suggests that the non zero value of $\left\langle u_{\ell}\right\rangle$ should mostly be due to a small fraction of informed trades, that correctly anticipate large price changes as a result of some private information, while most noise induced trades should only change the price on short time scales, before arbitrageurs set it back to its 'true' value. In this case, the positive tail of the distribution $P\left(u_{\ell}\right)$ (corresponding to informed trades) should be much fatter than the negative tail. This asymmetry can in fact 


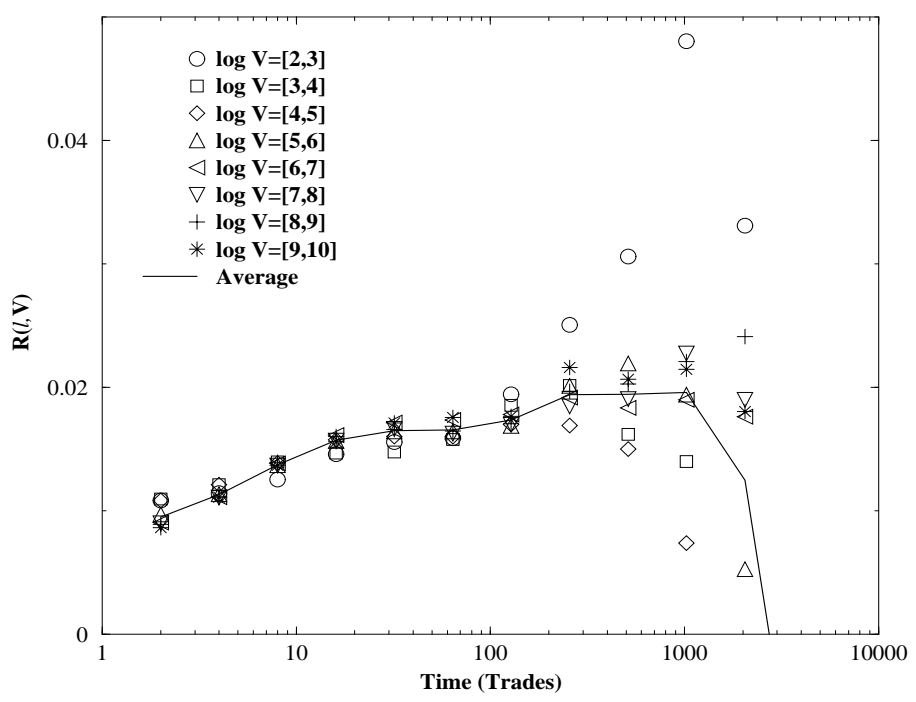

Figure 5: Average response function $\mathcal{R}(\ell, V)$, conditioned to a certain volume $V$, as a function of $\ell$. Data for different $V$ 's have been divided by $f(V) \propto \ln V$ such as to obtain good data collapse. The thick line corresponds to $\mathcal{R}(\ell)$ (unscaled).

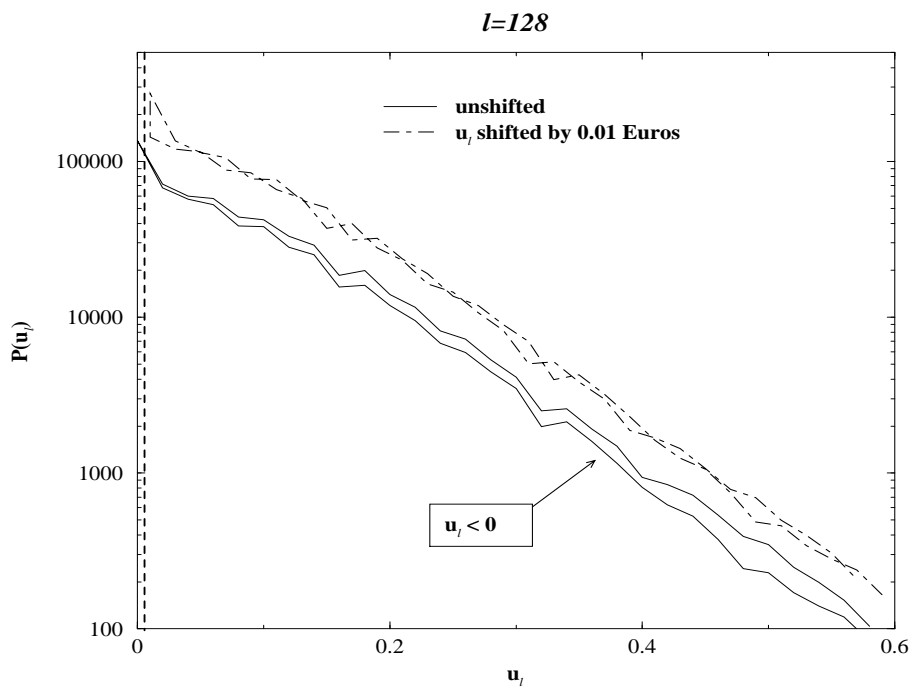

Figure 6: Probability distribution $P\left(u_{\ell}\right)$ of the quantity $u_{\ell}=\left(p_{n+\ell}-p_{n}\right) \cdot \varepsilon_{n}$ (in Euros), for $\ell=128$. The data is again FT during 2002. The negative part of the distribution has been folded back to positive $u_{\ell}$ in order to highlight the small positive skew of the distribution (which is seen to increase slightly with $\left|u_{\ell}\right|$ ). The average value $\mathcal{R}(\ell)=\left\langle u_{\ell}\right\rangle$ is shown as the vertical dashed line. The dasheddotted line corresponds to the distribution of $u_{\ell}-\nu$ with $\nu=0.01$ Euros. This curve has been shifted upwards for clarity. 
be taken as an objective measure of the fraction of informed trades. However, the nearly symmetric shape of $P\left(u_{\ell}-\nu\right)$ shown in Fig. 6 means that one can hardly detect the statistical presence of informed trades that correctly anticipate the sign of the price change on a short term basis, such as to at least cover their trading costs. $^{2}$ This result is consistent with the conclusion of other studies, where it is established that investors 'trade too much' 35], and that the uninformed price pressure is large [7. Note that $P\left(u_{\ell}\right)$ as defined above gives an equal weight to all trades, independently of their volume. We have also considered the volume weighted $P\left(u_{\ell}\right)$, which leads to the same qualitative conclusion.

The main conclusions of this section are thus that (a) large volumes impact prices on average much less (in relative terms) than smaller volumes, (b) the average impact of a given trade (as measured by $\mathcal{R}(\ell)$ ) increases with time up to a certain time scale $\ell^{*}$ beyond which it decreases and (c) the fraction of trades that correctly anticipates short term moves is small.

\subsection{A Fluctuation-Response relation}

In the study of Brownian particles, a very important result that dates back to Einstein relates the diffusion coefficient $D$ to the response of the particle to an external force. That a similar relation might also hold in financial markets was first suggested by Rosenow [36], and substantiated there by some empirical results. We have performed an analysis related to, but different from that of Rosenow. For any given trading day, one can compute the average local diffusion constant $\mathcal{D}(\ell)$ over a given time scale, say $\ell=128$, and the average local price response $\mathcal{R}(\ell)$ over the same time scale. Rosenow, on the other hand, computes a 'susceptibility' as the slope of the average price change over a given time interval versus the volume imbalance during the same time interval (see 32]), and relates this susceptibility to the diffusion constant. The analogue of Rosenow's result [36] (which was motivated by a Langevin equation for price variations - see [37]), is a linear relation between $\mathcal{R}^{2}(\ell)$ and $\mathcal{D}(\ell)$, which we illustrate in Fig. 7 for FT, for two different periods (first semester of 2001, and 2002). A similar result can also be read from Fig. 3. As will be clear in the following, such a relation will appear naturally within the simple model that we introduce in Section 3.

\subsection{Long term correlation of trade signs}

All the above results are compatible with a 'zero intelligence' picture of financial markets, where each trade is random in sign and shifts the price permanently, because all other participants update their evaluation of the stock price as a

\footnotetext{
${ }^{2}$ Some of these trades might of course be profitable on the long run. But since the price process is nearly diffusive and that the number of buy and sell market orders are nearly equal, it is clear that difference between the fraction of profitable trades on any given time scale and $50 \%$ is small.
} 


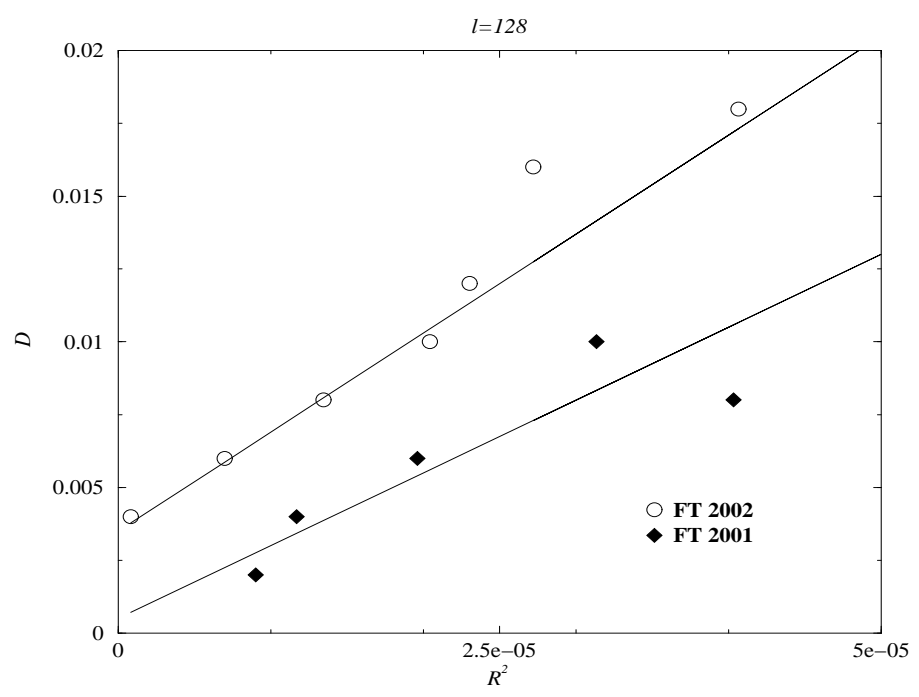

Figure 7: Average diffusion constant $D=\mathcal{D}(\ell) / \ell$, computed for $\ell=128$, and conditioned to a certain value of $\mathcal{R}^{2}(\ell)$, also computed for $\ell=128(\mathrm{FT})$. The open symbols correspond to 2002, whereas the black symbols are computed using the first semester of 2001, where the tick size was 5 times larger. Correspondingly, the $x$-axis was rescaled down by a factor 25 and the $y$-axis by a factor five for this data set.

function of the last trade. As shown in [9, 10, 12, 13, 14, 15], a model of the order book based on a purely random order flow indeed allows one to go quite far in the quantitative understanding of financial markets. In this context, the concave shape of the impact as a function of the volume can be understood as an order book effect, where the average size of the queue increases with depth. ${ }^{3}$

This model of a totally random stock market is however qualitatively incorrect for the following reason. Although, as mentioned above, the statistics of price changes reveals very little temporal correlations, the correlation function of the $\operatorname{sign} \varepsilon_{n}$ of the trades, on the other hand, reveals very slowly decaying correlations. This correlation has been mentioned in some papers before, see e.g. [7]. Here, we propose that these correlations decay as a power-law of the time lag.

More precisely, one can consider the following correlation function:

$$
\mathcal{C}_{0}(\ell)=\left\langle\varepsilon_{n+\ell} \varepsilon_{n}\right\rangle-\left\langle\varepsilon_{n}\right\rangle^{2}
$$

If trades were random, one should observe that $\mathcal{C}_{0}(\ell)$ decays to zero beyond a few trades. Surprisingly, this is not what happens: on the contrary, $\mathcal{C}_{0}(\ell)$ is strong

\footnotetext{
${ }^{3}$ However, other effects are probably important to understand this concavity, such as the conditioning of large market orders to the size of the order book - see [34, 7].
} 
and decays very slowly toward zero, as an inverse power-law of $\ell$ (see Fig. 8):

$$
\mathcal{C}_{0}(\ell) \simeq \frac{C_{0}}{\ell^{\gamma}}, \quad(\ell \geq 1)
$$

The value of $\gamma$ seems to be somewhat stock dependent. For example, for FT, one finds $\gamma \approx 1 / 5$, whereas for Total $\gamma \approx 2 / 3$. In their study, Lillo and Farmer found a somewhat larger value of $\gamma \approx 1 / 2$ for Vodafone [17. In any case, the value of $\gamma$

is found to be smaller than one, which is very important because the integral of $\mathcal{C}_{0}(\ell)$ is then divergent. Now, as will be shown more precisely in the next section, the integral of $\mathcal{C}_{0}(\ell)$ can intuitively be thought of as the effective number $N_{e}$ of correlated successive trades. Hence, out of - say - 1000 trades, one should group together

$$
N_{e} \simeq 1+\sum_{\ell=1}^{1000} \mathcal{C}_{0}(\ell) \approx 1+\frac{C_{0}}{1-\gamma} 1000^{1-\gamma}
$$

'coherent' trades. For FT, $\gamma \approx 1 / 5$ and $C_{0} \approx 0.2$, which means that the effect of one trade should be amplified, through the correlations, by a factor $N_{e} \approx 50$ ! In other words, both the response function $\mathcal{R}$ and the diffusion constant should increase by a factor 50 between $\ell=1$ and $\ell=1000$, in stark contrast with the observed empirical data. This is the main puzzle that one should try to elucidate: how can one reconcile the strong, slowly decaying correlations in the sign of the trades with the nearly diffusive nature of the price fluctuations, and the nearly structureless response function?

Before presenting a mathematical transcription of the above question and proposing a possible resolution, let us comment on two related correlation functions that will naturally appear in the following, namely:

$$
\mathcal{C}_{1}(\ell)=\left\langle\varepsilon_{n+\ell} \varepsilon_{n} \ln V_{n}\right\rangle,
$$

and

$$
\mathcal{C}_{2}(\ell)=\left\langle\varepsilon_{n+\ell} \ln V_{n+\ell} \varepsilon_{n} \ln V_{n}\right\rangle .
$$

We have found empirically that these two 'mixed' correlation functions are proportional to $\mathcal{C}_{0}(\ell)$ (see Fig 8 ):

$$
\mathcal{C}_{1}(\ell) \approx\langle\ln V\rangle \mathcal{C}_{0}(\ell) ; \quad \mathcal{C}_{2}(\ell) \approx\langle\ln V\rangle^{2} \mathcal{C}_{0}(\ell) .
$$

There are however small systematic deviations, which indicate that (i) small volumes contribute more to the long range correlations that larger volumes and (ii) $\ln V-\langle\ln V\rangle$ is a quantity exhibiting long range correlations as well.

\section{A micro-model of price fluctuations}

\subsection{Set up of the model}

In order to understand the above results, we will postulate the following trade superposition model, where the price at time $n$ is written as a sum over all past 


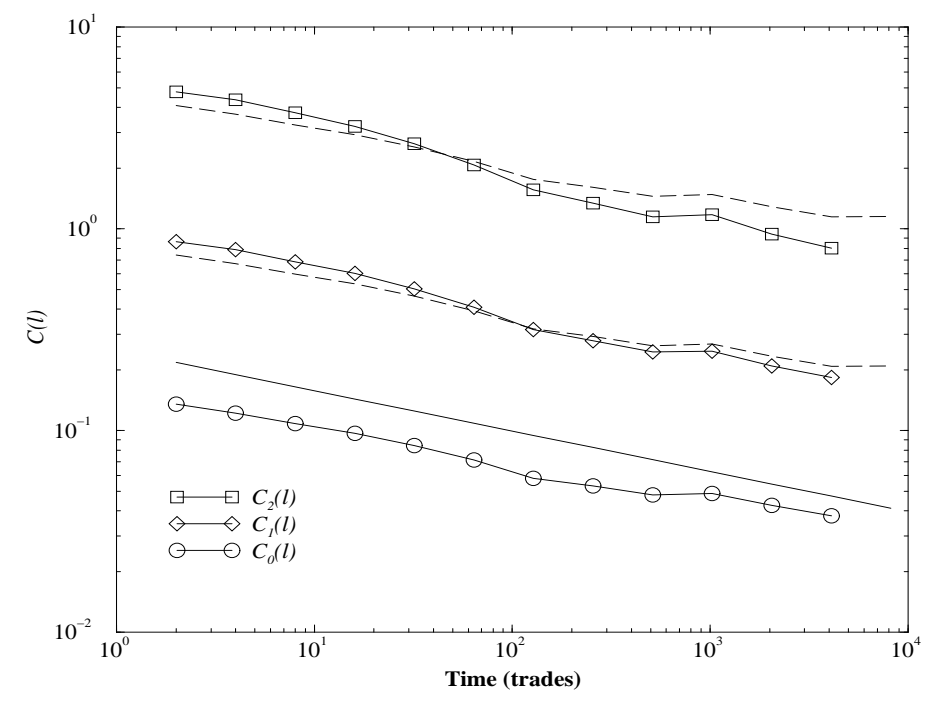

Figure 8: Volume weighted sign autocorrelation functions as a function of time lag: $\mathcal{C}_{0}, \mathcal{C}_{1}, \mathcal{C}_{2}$ (see text for definitions). The straight line corresponds to $\ell^{-\gamma}$ with $\gamma=1 / 5$. The dotted lines correspond to the simple approximation given by Eqs. (11).

trades, of the impact of one given trade propagated up to time $n$ :

$$
p_{n}=\sum_{n^{\prime}<n} G_{0}\left(n-n^{\prime}\right) \varepsilon_{n^{\prime}} \ln V_{n^{\prime}}+\sum_{n^{\prime}<n} \eta_{n^{\prime}}
$$

where $G_{0}($.$) is the 'bare' impact function (or propagator) of a single trade, that we$ assume to be a fixed, non random function that only depends on time differences. The $\eta_{n}$ are also random variables, assumed to be independent from the $\varepsilon_{n}$ and model all sources of price changes not described by the direct impact of the trades: the bid-ask can change as the result of some news, or of some order flow, in the absence of any trades. We will in the following assume that the $\eta_{n}$ are also uncorrelated in time, although this assumption can easily be relaxed. In the above model, we assume that the 'bare' impact function $G_{0}$ is not itself fluctuating, which can only be an approximation.

The bare impact function $G_{0}(\ell)$ represents by definition the average impact of a single trade after $\ell$ trades. It could be in principle measured empirically by launching on the market a sequence of real trades of totally random signs, and averaging the impact over this sample of trades (a potentially costly experiment!). ${ }^{4}$ As will be clear below, the difference between the quantity $\mathcal{R}(\ell)$ introduced in the previous Section and $G_{0}(\ell)$ in fact comes from the strong autocorrelation of the sign of the trades. In order to understand the temporal structure of $G_{0}(\ell)$,

\footnotetext{
${ }^{4}$ However, following this procedure might induce 'copy-cat' trades and still lead to a difference between the measured response function and $G_{0}$
} 
note that a single trade first impacts the midpoint by changing the bid (or the ask). But then the subsequent limit order flow due to that particular trade might either center on average around the new midpoint (in which case $G_{0}(\ell)$ would be constant), or, as we will argue below, tend to mean revert toward the previous midpoint (in which case $G_{0}(\ell)$ decays with $\ell$ ). As discussed below (see Eq. (26) ), the asymptotic behaviour of the bare impact function in fact reveals the average cost of a single market order: if $G_{0}(\ell \gg 1) / G_{0}(1)$ is small, the cost is large since the initial impact of the trade is only temporary, and is not followed by a true long term change of the price.

Using this representation, the price increment between an arbitrarily chosen initial time 0 and time $\ell$ is:

$$
p_{\ell}-p_{0}=\sum_{0 \leq n<\ell} G_{0}(\ell-n) \varepsilon_{n} \ln V_{n}+\sum_{n<0}\left[G_{0}(\ell-n)-G_{0}(-n)\right] \varepsilon_{n} \ln V_{n}+\sum_{0 \leq n<\ell} \eta_{n} .
$$

If the signs $\varepsilon_{n}$ were independent random variables, both the response function and the diffusion would be very easy to compute. For example, one would have: ${ }^{5}$

$$
\mathcal{R}_{t}(\ell)=\langle\ln V\rangle G_{0}(\ell)
$$

i.e. the observed impact function and the bare response function would be proportional. Similarly, one would have:

$$
\mathcal{D}_{t}(\ell)=\left\langle\ln ^{2} V\right\rangle\left(\sum_{0<n \leq \ell} G_{0}^{2}(n)+\sum_{n>0}\left[G_{0}(\ell+n)-G_{0}(n)\right]^{2}\right)+D_{\eta} \ell,
$$

where $D_{\eta}$ is the variance of the $\eta$ 's. In the simplest case of a constant bare impact function, $G_{0}(\ell)=\Gamma_{0}$ for all $\ell>0$, one then finds a pure diffusive behaviour, as expected:

$$
\mathcal{D}_{t}(\ell)=\ell\left[\left\langle\ln ^{2} V\right\rangle \Gamma_{0}^{2}+D_{\eta}\right] .
$$

This result (no correlations between the $\varepsilon$ 's and a constant bare impact function) corresponds to the simplest possible zero intelligence market. However, we have seen that in fact the $\varepsilon$ 's have long range correlations. In this case, the average response function reads:

$$
\mathcal{R}_{t}(\ell)=\langle\ln V\rangle G_{0}(\ell)+\sum_{0<n<\ell} G_{0}(\ell-n) \mathcal{C}_{1}(n)+\sum_{n>0}\left[G_{0}(\ell+n)-G_{0}(n)\right] \mathcal{C}_{1}(n) .
$$

Note in passing that our trade superposition model, Eq. (12), together with Eq. (11) leads to the factorization property mentioned above (see Fig. 5):

$$
\mathcal{R}_{t}(\ell, V)=\frac{\ln V}{\langle\ln V\rangle} \mathcal{R}_{t}(\ell)
$$

\footnotetext{
${ }^{5}$ In the following, we will use the subscript ' $t$ ' to denote the theoretical expressions for the response function or diffusion.
} 
Now, one sees more formally the paradox discussed in the previous Section: assuming that the impact of each trade is permanent, i.e. $G_{0}(\ell)=\Gamma_{0}$, leads to:

$$
\mathcal{R}_{t}(\ell)=\Gamma_{0}\left[\langle\ln V\rangle+\sum_{0<n<\ell} \mathcal{C}_{1}(n)\right]
$$

If $\mathcal{C}_{1}(n)$ decays as a power-law with an exponent $\gamma<1$, then the average impact $\mathcal{R}(\ell)$ should grow like $\ell^{1-\gamma}$, and therefore be amplified by a very large factor as $\ell$ increases, at variance with empirical data. The only way out of this conundrum is (within the proposed model) that the bare impact function $G_{0}(\ell)$ itself should decay with time, in such a way to offset the amplification effect due to the trade correlations.

\subsection{A relation between the bare propagator and the sign correlation function}

In order to get some guidance, let us now look at the general formula for the diffusion. After a few lines of calculations, one finds:

$$
\begin{aligned}
\mathcal{D}_{t}(\ell) & =\left\langle\ln ^{2} V\right\rangle\left[\sum_{0 \leq n<\ell} G_{0}^{2}(\ell-n)+\sum_{n>0}\left[G_{0}(\ell+n)-G_{0}(n)\right]^{2}\right] \\
& +2 \Delta(\ell)+D_{\eta} \ell,
\end{aligned}
$$

where $\Delta(\ell)$ is the correlation induced contribution:

$$
\begin{aligned}
\Delta(\ell) & =\sum_{0 \leq n<n^{\prime}<\ell} G_{0}(\ell-n) G_{0}\left(\ell-n^{\prime}\right) \mathcal{C}_{2}\left(n^{\prime}-n\right) \\
& +\sum_{0<n<n^{\prime}}\left[G_{0}(\ell+n)-G_{0}(n)\right]\left[G_{0}\left(\ell+n^{\prime}\right)-G_{0}\left(n^{\prime}\right)\right] \mathcal{C}_{2}\left(n^{\prime}-n\right) \\
& +\sum_{0 \leq n<\ell} \sum_{n^{\prime}>0} G_{0}(\ell-n)\left[G_{0}\left(\ell+n^{\prime}\right)-G_{0}\left(n^{\prime}\right)\right] \mathcal{C}_{2}\left(n^{\prime}+n\right)
\end{aligned}
$$

The constraint from empirical data is that this expression must be approximately linear in $\ell$. As shown in the Appendix, the requirement that $\mathcal{D}_{t}(\ell)$ is strictly linear in $\ell$ for all $\ell$ in fact allows one to express $G_{0}(\ell)$ as a function of $\mathcal{C}_{2}(\ell)$. Here, we present a simple asymptotic argument. If we make the ansatz that the bare impact function $G_{0}(\ell)$ also decays as a power-law:

$$
G_{0}(\ell)=\frac{\Gamma_{0} \ell_{0}^{\beta}}{\left(\ell_{0}+\ell\right)^{\beta}} \quad(\ell \geq 1)
$$

then one can estimate $\mathcal{D}_{t}(\ell)$ in the large $\ell$ limit. When $\gamma<1$, one again finds that the correlation induced term $\Delta(\ell)$ is dominant, and all three terms scale a $\ell^{2-2 \beta-\gamma}$, provided $\beta<1$. In other words, the Hurst exponent of price changes 
is given by $2 H=2-2 \beta-\gamma$. Therefore, the condition that the fluctuations are diffusive at long times $(H=1 / 2)$ imposes a relation between the decay of the sign autocorrelation $\gamma$ and the decay of the bare impact function $\beta$ that reads:

$$
2 \beta+\gamma=1 \longrightarrow \beta_{c}=\frac{1-\gamma}{2}
$$

For $\beta>\beta_{c}$, the price is sub-diffusive $(H<1 / 2)$, which means that price changes show anti-persistence; while for $\beta<\beta_{c}$, the price is super-diffusive $(H>1 / 2)$, i.e. price changes are persistent. For FT, $\gamma \approx 1 / 5$ and therefore $\beta_{c} \approx 2 / 5$.

As shown in the Appendix, one can in fact obtain an exact relation between $G_{0}(\ell)$ and $\mathcal{C}_{2}(\ell)$ if one assumes that price changes are strictly uncorrelated (i.e. that $\mathcal{D}(\ell)$ is linear in $\ell$ for all $\ell$ ). The asymptotic analysis of this relation leads, not surprisingly, to the same exponent relation $\beta_{c}=(1-\gamma) / 2$ as above.

At this stage, there seems still to be a contradiction with empirical data, for if one goes back to the response function given by Eq. (17), one finds that whenever $\beta+\gamma<1$ (which is indeed the case for $\beta=\beta_{c}$ and $\gamma<1$ ), the dominant contribution to $\mathcal{R}_{t}(\ell)$ should behave as $\ell^{1-\beta-\gamma}$ and thus grow with $\ell$. For example, for $\gamma \approx 1 / 5$ and $\beta \approx 2 / 5$, one should find that $\mathcal{R}_{t}(\ell) \propto \ell^{2 / 5}$, which is incompatible with the empirical data of Figs. 3 and 4 . But the surprise comes from the numerical prefactor of this power law. One finds, for large $\ell$ :

$$
\mathcal{R}_{t}(\ell) \simeq\langle\ln V\rangle \Gamma_{0} C_{0} \frac{\Gamma(1-\gamma)}{\Gamma(\beta) \Gamma(2-\beta-\gamma)}\left[\frac{\pi}{\sin \pi \beta}-\frac{\pi}{\sin \pi(1-\beta-\gamma)}\right] \ell^{1-\beta-\gamma} .
$$

Therefore, only when $\beta=\beta_{c}$, is the prefactor exactly zero, and leads to the possibility of a nearly constant impact function! For faster decaying impact functions (larger $\beta$ 's), this prefactor is negative, whereas for more slowly decaying impact functions this prefactor is positive. ${ }^{6}$ Interestingly, even if the bare response function $G_{0}(\ell)$ is positive for all $\ell$, the average response $\mathcal{R}_{t}(\ell)$ can become negative for large enough $\beta$ 's, as a consequence of the correlations between trades.

\subsection{Fitting the average response function}

Since the dominant term is zero for the 'critical' case $\beta=\beta_{c}$, and since we are interested in the whole function $\mathcal{R}_{t}(\ell)$ (including the small $\ell$ regime), we have computed $\mathcal{R}_{t}(\ell)$ numerically, by performing the discrete sum Eq. (17) exactly, and fitted it to the empirical response $\mathcal{R}$. The results are shown in Fig. 9. We have fixed the parameters $\gamma$ and $C_{0}$ to the values extracted from the behaviour of $\mathcal{C}_{1}(\ell)$ (see Fig. 8): $\gamma=0.24$ and $C_{0}=0.20$. The overall scaling parameter $\Gamma_{0}$ is adjusted to $\Gamma_{0}=2.810^{-3}$ Euros to match the value of $\mathcal{R}(\ell=1)$. The values of $\beta$

\footnotetext{
${ }^{6}$ Note that although this prefactor increases (in absolute value) with $\beta$ for $\beta>\beta_{c}$, the power of $\ell$ decreases, which means that for large $\ell$ the amplitude of $\mathcal{R}_{t}(\ell)$ decreases with $\beta$, as intuitively expected.
} 


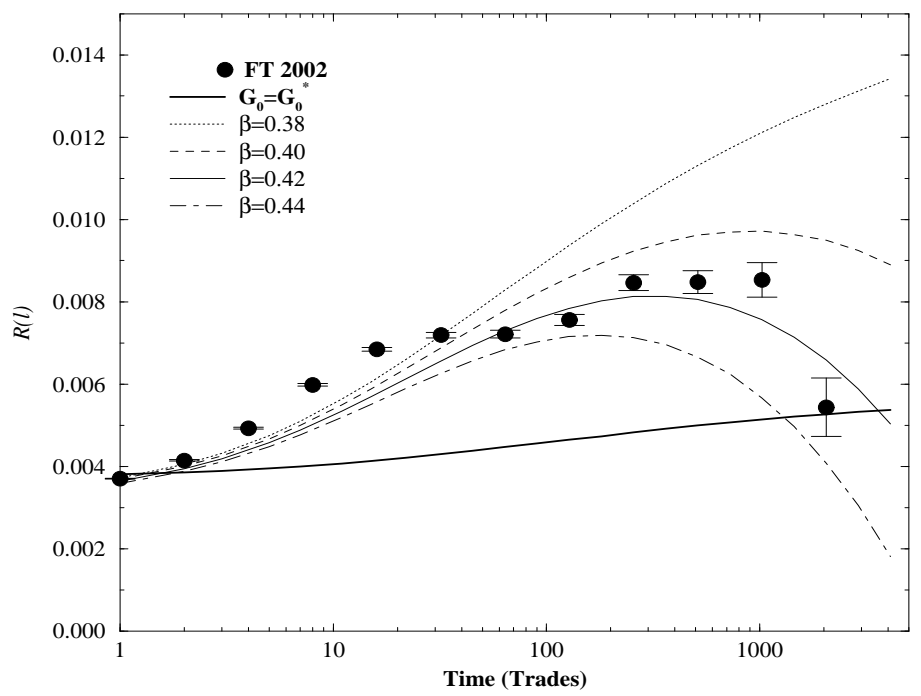

Figure 9: Theoretical impact function $\mathcal{R}_{t}(\ell)$, from Eq. (17), and for different values of $\beta$ close to $\beta_{c}=0.38$. The shape of the empirical response function can be quite accurately reproduced using $\beta=0.42$. The only remaining free parameter is $\ell_{0}=20$. The thick plain line is $\mathcal{R}_{t}(\ell)$ computed using the "pure diffusion' propagator $G_{0}^{*}$ determined in Appendix, Eq. (34).

and $\ell_{0}$ are fitting parameters: we show in Fig. 9 the response function computed for different values of $\beta$ in the vicinity of $\beta_{c}=0.38$, and used $\ell_{0}=20$.

The results are compared with the empirical data for FT, showing that one can indeed satisfactorily reproduce, when $\beta \approx \beta_{c}$, a weakly increasing impact function that reaches a maximum and then decays. One also sees, from Fig. 9, that the relation between $\beta$ and $\gamma$ must be quite accurately satisfied, otherwise the response function shows a distinct upward trend (for $\beta<\beta_{c}$ ) or a downward trend $\left(\beta>\beta_{c}\right) .^{7}$ In fact, we have tried other simple forms for $G_{0}(\ell)$, such as a simple exponential decay toward a possibly non zero asymptotic value, but this leads to unacceptable shapes for $\mathcal{R}(\ell)$.

It is also interesting to use the propagator $G_{0}^{*}$ determined in the Appendix from the assumption of a purely diffusive price process for all $\ell$ 's. This propagator is plotted in Fig. 10, and compared to the $G_{0}$ determined above from the fit of $\mathcal{R}(\ell)$. As shown in Fig. 9, the use of $G_{0}^{*}$ does not lead to a very good fit of $\mathcal{R}(\ell)$. Since the latter quantity is in fact very sensitive to the chosen shape for $G_{0}$, it does reveal small, but systematic deviations from a purely diffusive price process. [Note that if one had $\mathcal{C}_{2}(\ell)=\mathcal{C}_{1}(\ell)$, the resulting $\mathcal{R}(\ell)$ should be strictly constant.]

\footnotetext{
${ }^{7}$ This might actually explain the different behaviour of Pechiney seen in Fig. 4.
} 


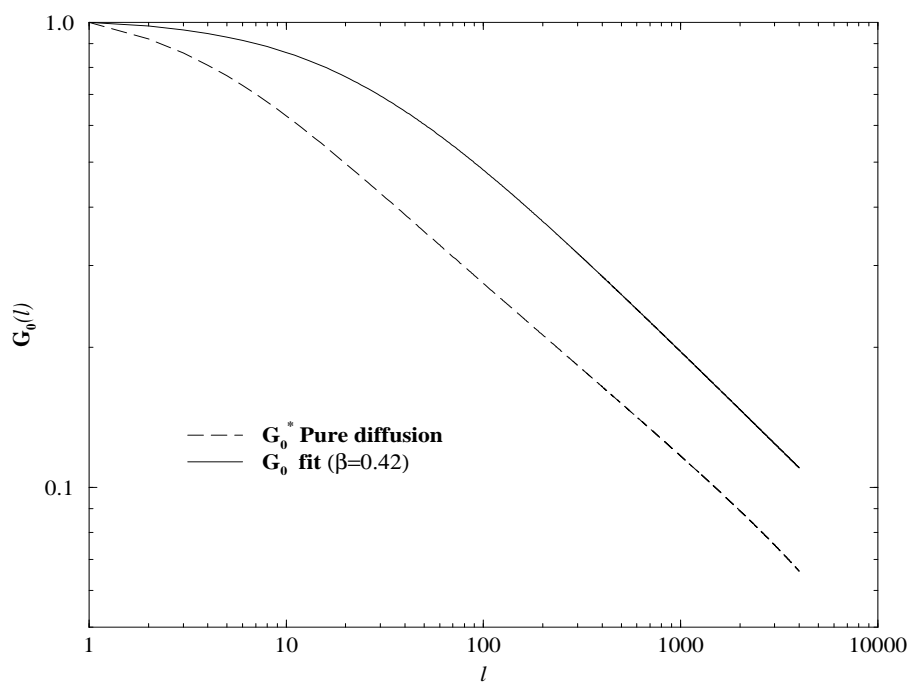

Figure 10: Shape of the bare propagator $G_{0}$, determined either by the fit of $\mathcal{R}$, with $\beta=0.42$ and $\ell_{0}=20$, or using the exact relation, Eq. (34), derived in the Appendix from the assumption of a purely diffusive process.

\subsection{Back to the diffusion constant}

As we showed above, the reason for the fine tuning of $\beta$ is the requirement that price changes are almost diffusive. We can therefore also compute $\mathcal{D}_{t}(\ell)$ for all values of $\ell$ using the very same values of $\gamma, \beta, C_{0}, \ell_{0}$ and $\Gamma_{0}$. Now, in order to fit the data one has two extra free parameters: one is $D_{\eta}$, and the other comes about because the mid-point can change without any trade. One should thus add to $\mathcal{D}_{t}(\ell)$ an $\ell$-independent 'error' term $D_{0}$ that survives in the $\ell=0$ limit, and is associated to bid-ask fluctuations. With these two extra parameters, one can reproduce the empirical determination of $\mathcal{D}(\ell) / \ell$ (see Fig. 11). The small deviations of this quantity from a horizontal line at finite $\ell$ are due to the difference between $G_{0}$ and $G_{0}^{*}$ and/or to the possible autocorrelations between the $\eta_{n}$ variables, which we have neglected here. Note that the contribution of the term $D_{\eta}$ turns out to be a factor two larger than that of the impact contribution, Eq. (20), which means that the small increase of the 'impact contribution' with $\ell$ (lower graph of Fig. 11) is hardly detectable in $\mathcal{D}(\ell) / \ell$.

Coming back to the Fluctuation-Response relation discussed in Section 2.4, we see that our model predicts, for $\ell \gg 1$ where the effect of $D_{0}$ can be neglected:

$$
\frac{\mathcal{D}_{t}(\ell)}{\ell}=Z\langle\ln V\rangle^{2} C_{0} \Gamma_{0}^{2}+D_{\eta}, \quad \mathcal{R}_{t}(\ell)=Z^{\prime}\langle\ln V\rangle \Gamma_{0} C_{0},
$$

where $Z, Z^{\prime}$ are numerical constants. Assuming that from one day to the next both the average (log-)traded volume and the impact $\Gamma_{0}$ of each individual trade 


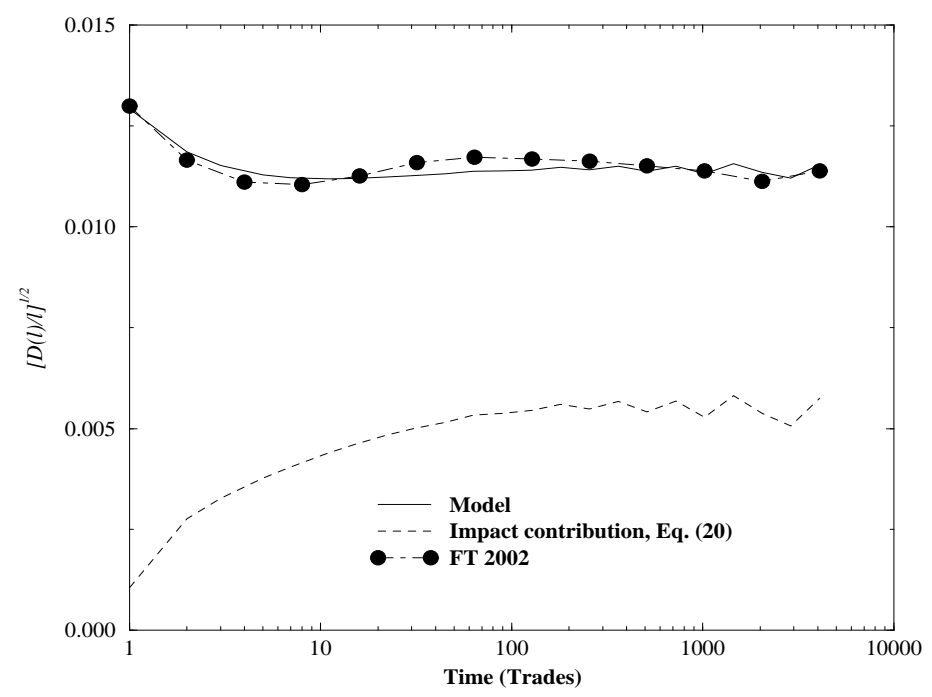

Figure 11: Diffusion constant $\mathcal{D}(\ell) / \ell$, using Eq. (20), with the values of $\gamma, \beta, C_{0}$, $\ell_{0}$ and $\Gamma_{0}$ determined from $\mathcal{R}(\ell)$. Two extra parameters were used: $D_{\eta}=10^{-4}$ and $D_{0}=6.610^{-5}$ (both in Euro squared). The lower graph is the 'impact contribution' to $\mathcal{D}_{t}(\ell)$, given by Eq. (20) with $D_{\eta}=0$. The 'oscillations' at long times is a numerical artefact.

might change, while $C_{0}$ is fixed, immediately leads to the affine relation between $\mathcal{D}$ and $\mathcal{R}^{2}$ reported in Section 2.4.

\subsection{Discussion}

The conclusion of this Section is that our 'micro-model' of prices, Eq. (12), can be used as a theoretical canvas to rationalize and interpret the empirical results found in the previous Section. Most surprising is the constraint that the empirical results impose on the shape of the 'bare' response function $G_{0}$, which is found to be a slowly decaying power law which must precisely cancel the slowly decaying autocorrelation of the trades, but reveals systematic deviations from a pure diffusion process, hardly noticeable on the diffusion constant itself. The fact that the bare impact function decays with time (at least on intra-day time scales), in a finely tuned way to compensate the long memory in the trades, is the central result of this paper. This effect is lost in the zero intelligence models of Poisonnian order flows, where, after decreasing during a short transient, the impact of each trade becomes permanent: $G_{0}(\ell) \rightarrow G_{\infty}>0$. (On this point, see the model studied in [14, where it is shown that prices are sub-diffusive on time scales shorter than the life-time of limit orders, essentially as a consequence of the shape of the order book). In fact, both the long time memory of the trades and the slowly relaxing impact function reported here must be the consequence 
of the strategic behaviour of market participants, that we discuss below in order to get an intuitive understanding of the mechanisms at play.

Although our detailed analysis concerns FT, it is clear that our conclusions are more general, since both the strong autocorrelations in the trade signs, the near constancy of the average response function and the diffusive nature of price changes have been observed on all stocks, with only quantitative changes (see Figs 2 and 4). It would be interesting to document these quantitative difference, and relate these to liquidity, or to the size of the bid-ask spread.

Finally, it would be very interesting to know whether the bare response function levels off to a finite value for large time lags; this will require to go beyond the analysis of the present paper and to deal with overnight effects to enlarge the available range of $\ell$ values. However, it seems reasonable to expect that $G_{0}(\ell)$ should indeed reach a finite asymptotic value for values of $\ell$ corresponding to a few days of trading. ${ }^{8}$

\section{Critical balance of opposite forces: Market orders vs. limit orders}

Although trading occurs for a large variety of reasons, it is useful to recognize that traders organize in two broad categories:

- One is that of 'liquidity takers', that trigger trades by putting in market orders. The motivation for this category of traders might be to take advantage of some 'information', and make a profit from correctly anticipating future price changes. Information can in fact be of very different nature: fundamental (firm based), macro-economical, political, statistical (based on regularities of price patterns), etc. Unfortunately, information is often hard to interpret correctly, and it is probable that many of these "information' driven trades are misguided (on this point, see the remarkable work of Odean [35]; see also [7] and refs. therein). For example, systematic hedge funds which take decisions based on statistical pattern recognition have a typical success rate of only $52 \%$. There is no compelling reason to believe that the intuition of traders in markets room fares much better than that. Since market orders allows one to be immediately executed, many impatient investors, who want to liquidate their position, or hedge, etc. might be tempted to place market orders, even at the expense of the bid-ask spread $s(t)=a(t)-b(t)$.

- The other category is that of 'liquidity providers' (or 'market makers', although on electronic markets all participants can act as liquidity providers

\footnotetext{
${ }^{8}$ Hopman quotes three days as the time beyond which the autocorrelation of the trades sign falls to zero [7].
} 
by putting in limit orders), who offer to buy or to sell but avoid taking any bare position on the market. Their profit comes from the bid-ask spread $s$ : the sell price is always slightly larger than the buy price, so that each round turn operation leads to a profit equal to the spread $s$, at least if the midpoint has not changed in the mean time (see below).

This is where the game becomes interesting. Assume that a liquidity taker wants to buy, so that an increased number of buy orders arrive on the market. The liquidity providers is tempted to increase the offer (or ask) price $a$ because the buyer might be informed and really know that the current price is too low and that it will most probably increase in the near future. Should this happen, the liquidity provider, who has to close his position later, might have to buy back at a much higher price and experience a loss. In order not to trigger a sudden increase of $a$ that would make their trade costly, liquidity takers obviously need to put on not too large orders. This is the rationale for dividing one's order in small chunks and disperse these as much as possible over time so as not to appear on the 'radar screens'. Doing so liquidity takers necessarily create some temporal correlations in the sign of the trades. Since these traders probably have a somewhat broad spectrum of volumes to trade [38, and therefore of trading horizons (from a few minutes to several weeks), this can easily explain the slow, power-law decay of the sign correlation function $\mathcal{C}_{0}(\ell)$ reported above.

Now, if the market orders in fact do not contain useful information but are the result of hedging, noise trading, misguided interpretations, errors, etc., then the price should not move up on the long run, and should eventually mean revert to its previous value. Liquidity providers are obviously the active force behind this mean reversion, again because closing their position will be costly if the price has moved up too far from the initial price. More precisely, a computation of the liquidity provider average gain per share $\mathcal{G}$ can be performed [16], and is found to be, for trades of volume $V$ :

$$
\mathcal{G}=s+\mathcal{R}(0, V)-\mathcal{R}(\infty, V) \approx s+\ln V[\mathcal{R}(0)-\mathcal{R}(\infty)],
$$

where $\mathcal{R}(0, V)$ is the immediate average impact of a trade, before new limit orders set in. We have in fact checked empirically that $\mathcal{R}(0, V) \approx \mathcal{R}(1, V)$. From the above formula, one sees that it is in the interest of liquidity providers to mean revert the price, such as to make $\mathcal{R}(\infty)$ as small as possible. However, this mean reversion cannot take place too quickly, again because a really informed trader would then be able to buy a large volume at a modest price. Hence, this mean reversion must be slow. From the quantitative analysis of Section 3, we have found that there is hardly any mean reversion at all on short time scales $\ell<\ell_{0}$, and that this effect can be described as a slow power-law for larger $\ell$ 's. Actually, the action of liquidity providers and liquidity takers must be such that no (or very little) linear correlation is created in the price changes, otherwise statistical 
arbitrage opportunities would be created at the detriment of one or the other population.

To summarize: liquidity takers must dilute their orders and create long range correlations in the trade signs, whereas liquidity providers must correctly handle the fact that liquidity takers might either possess useful information (a rare situation, but that can be very costly since the price can jump as a result of some significant news), or might not be informed at all and trade randomly. By slowly mean reverting the price, market makers minimize the probability that they either sell too low, or have to buy back too high. The delicate balance between these conflicting tendencies conspire to put the market at the border between persistence (if mean reversion is too weak, i.e. $\beta<\beta_{c}$ ) or anti-persistence (if mean reversion is too strong, i.e. $\beta>\beta_{c}$ ), and therefore eliminate arbitrage opportunities.

It is actually enlightening to propose a simple model that could explain how market makers enforce this mean reversion. ${ }^{9}$ Assume that upon placing limit orders, there is a systematic bias toward some moving average of past prices. If this average is for simplicity taken to be an exponential moving average, the continuous time description of this will read:

$$
\begin{aligned}
\frac{d p_{t}}{d t} & =-\Omega\left(p_{t}-\bar{p}_{t}\right)+\eta_{t} \\
\frac{d \bar{p}_{t}}{d t} & =\kappa\left(p_{t}-\bar{p}_{t}\right),
\end{aligned}
$$

where $\eta_{t}$ is the random driving force due to trading, $\Omega$ the inverse time scale for the strength of the mean reversion, and $1 / \kappa$ the 'memory' time over which the average price $\bar{p}_{t}$ is computed. The first equation means that liquidity providers tend to mean revert the price toward $\bar{p}_{t}$, while the second describes the update of the exponential moving average $\bar{p}_{t}$ with time. This set of linear equations can be solved, and leads to a solution of the form $p_{t}=\int^{t} d t^{\prime} G_{0}\left(t-t^{\prime}\right) \eta_{t}^{\prime}$, with a bare propagator given by:

$$
G_{0}(t)=\left(1-G_{\infty}\right) \exp [-(\Omega+\kappa) t]+G_{\infty},
$$

i.e. an exponential decay toward a finite asymptotic value $G_{\infty}=\kappa /(\Omega+\kappa)$. Note that, interestingly, it is the self-referential effect that leads to a non zero asymptotic impact. If the fundamental price was known to all, $\kappa=0$ and $G_{\infty}=0$. In the opposite limit where $\kappa \gg \Omega$, the last price is taken as the reference price, and $G_{\infty} \rightarrow 1$. A way to obtain $G_{0}(t)$ to resemble a power-law is to assume that different market makers use different time horizons to compute a reasonable reference price. This leads to a $G_{0}(t)$ which writes as the sum of time exponentials with different rates which can easily mimic a pure power-law.

\footnotetext{
${ }^{9}$ We have in fact directly checked on the data that the evolution of the midpoint between trades (resulting from the order flow) is indeed anticorrelated with the impact of the trades. On this point, see also [7] where the limit order flow subsequent to a trade is studied.
} 
The message of the above model is actually quite interesting from the point of view of Efficient Markets: it suggests that nobody really knows what the correct reference price should be, and that its best proxy is in fact its own past average over some time window (the length of which being itself distributed over several time scales).

\section{Summary and Conclusion}

The aim of this paper was to study in details the statistics of price changes at the trade by trade level, and to analyze the interplay between the impact of each trade on the price and the volatility. Empirical data shows that (a) the price (midpoint) process is close to being purely diffusive, even at the trade by trade scale (b) the temporal structure of the impact function first increases and reaches a maximum after 100 - 1000 trades, before decreasing back, with a rather limited overall variation (typically a factor 2) and (c) the sign of the trades shows surprisingly long range (power-law) correlations. The paradox is that if the impact of each trade was permanent, the price process should be strongly super-diffusive and the average response function should increase by a large factor as a function of the time-lag.

As a possible resolution of this paradox, we have proposed a micro-model of prices, Eq. (12) where the price at any instant is the causal result of all past trades, mediated by what we called a bare impact function, or propagator $G_{0}$. All the empirical results can be reconciled if one assumes that this bare propagator also decays as a power-law in time, with an exponent which is precisely tuned to a critical value, ensuring simultaneously that prices are diffusive on long time scales and that the response function is nearly constant. Therefore, the seemingly trivial random walk behaviour of price changes in fact results from a fined-tuned competition between two opposite effects, one leading to superdiffusion (the autocorrelation of trades) and the other leading to sub-diffusion (the decay of the bare impact function). The cancellation is however not exact: the non trivial behaviour of the average response function allows one to detect small, but systematic deviations from a purely diffusive behaviour, deviations that are hardly detectable on the price fluctuations themselves.

In financial terms, the competition is between liquidity takers, that create long range correlations by dividing their trading volume in small quantities, and liquidity providers that tend to mean revert the price such as to optimize their

gains (see Eq. (26) ). The resulting absence of correlations in price changes, and therefore of arbitrage opportunities is often postulated a priori in the economics literature, but the details of the mechanism that removes these arbitrage opportunities are rather obscure. The main message of this paper is that the random walk nature of price changes is not due to the unpredictable nature of incoming news, but appears as a dynamical consequence of the competition between an- 
tagonist market forces. In fact, the role of real (and correctly interpreted) news appears to be rather thin: we have defined a model independent indicator of the fraction of 'informed' trades, as the asymmetry of the probability distribution of the signed price variation, where the sign is that of the trade at the initial time. Information triggered trades should reveal in a detectable positive skew of this distribution, in particular in the tails. Consistently with other studies [35], our empirical results only show very weak asymmetry, barely sufficient to cover trading costs, which means that only a small fraction of trades can a posteriori described as truly informed, whereas most trades can be classified as noise. This result is most probably one of the mechanism needed to explain the excess volatility puzzle first raised by Schiller [3].

From a more general standpoint, our finding that the absence of arbitrage opportunities results from a critical balance between antagonist effects is quite interesting. It might justify several claims made in the (econo-)physics literature that the anomalies in price statistics (fat tails in returns described by power laws [20, 21, long range self similar volatility correlations [24, 25], and the long ranged correlations in signs reported here and in [17) are due to the presence of a critical point in the vicinity of which the market operates (see e.g. [39], and in the context of financial markets [40, 41]). If a fine-tuned balance between two competing effects is needed to ensure absence of arbitrage opportunities, one should expect that fluctuations are crucial, since a local unbalance between the competing forces can lead to an instability. In this respect, the analogy with the balancing of a long stick is quite enticing [19]. In more financial terms, the breakdown of the conditions for this dynamical equilibrium is, for example, a liquidity crisis: a sudden cooperativity of market orders, that lead to an increase of the trade sign correlation function, can out-weight the liquidity providers stabilizing (mean-reverting) role, and lead to crashes. This suggests that one should be able to write a mathematical model, inspired by our results, to describe this 'on-off intermittency' scenario, advocated (although in a different context) in [19, 42, 43].

Acknowledgments: Yuval Gefen thanks Science \& Finance/Capital Fund Management for hospitality during the period this work was completed. We thank Jelle Boersma, Lisa Borland and Bernd Rosenow for inspiring remarks, and J. Doyne Farmer for communicating his results on the autocorrelation of trades before publication [17] and for insightful discussions and comments. We also thanks S. Picozzi for an interesting discussion and for pointing out the possible relevance of ref. [19] to financial markets. Xavier Gabaix has made some crucial remarks on the first version of the manuscript, that in particular lead to the material contained in the Appendix, and draw our attention to ref. [7. Finally, we take the opportunity of this paper to acknowledge the countless efforts of Gene Stanley to investigate the dynamics of complex systems and to bring together different fields and ideas - as testified by the papers cited in reference, which inspired the present work. 


\section{Appendix: The case of a strictly diffusive process}

This appendix was inspired by a remark of Xavier Gabaix. There is one particular case of our micro-model of prices, Eq. (12), where prices are purely diffusive at all times (rather than only asymptotically). This is the case provided a specific relation between the bare propagator $G_{0}$ and the sign correlation function $\mathcal{C}_{2}(\ell)$ holds. In order to show this, let us assume that the random variable $q_{n} \equiv \varepsilon_{n} \ln V_{n}$ can be written as:

$$
q_{n}=\sum_{m \leq n} K(n-m) \xi_{m},
$$

where $\xi_{n}$ are uncorrelated random variables $\left(\left\langle\xi_{n} \xi_{m}\right\rangle=\left\langle\ln ^{2} V\right\rangle \delta_{n, m}\right)$, and $K($.$) a$ certain kernel. In order for the $q_{n}$ to have the required correlations, the kernel $K($.$) should obey the following equation:$

$$
\mathcal{C}_{2}(n)=\left\langle\ln ^{2} V\right\rangle \sum_{m \geq 0} K(m+n) K(m) .
$$

In the case where $\mathcal{C}_{2}$ decays as $\ell^{-\gamma}$ with $0<\gamma<1$, it is easy to show that the asymptotic decay of $K(n)$ should also be a power-law $n^{-\delta}$ with $2 \delta-1=\gamma$. Note that $1 / 2<\delta<1$.

Inverting Eq. (29) allows one to obtain a set of uncorrelated random variables $\xi_{n}$ from a set of correlated variables $q_{n}$ :

$$
\xi_{n}=\sum_{m \leq n} Q(n-m) q_{m}
$$

where $Q$ is the matrix inverse of $K$, such that $\sum_{m=0}^{n} K(n-m) Q(m)=\delta_{m, n}$. Eqs. (2931) in fact form the basis of linear filter theories, and $\xi_{n}$ can be seen as the prediction error on the next variable $q_{n}$.

Introducing discrete Laplace transforms:

$$
\widehat{K}(E)=\sum_{n \geq 0} K(n) e^{-n E} \quad \widehat{Q}(E)=\sum_{n \geq 0} Q(n) e^{-n E},
$$

one finds $\widehat{K}(E) \widehat{Q}(E)=1$. For a power-law kernel $K($.), one obtains: $\widehat{Q}(E) \propto$ $E^{1-\delta}$ for $E \rightarrow 0$, and therefore $Q(n) \propto n^{\delta-2}$ for large $n$. It is useful to note that in this case $\widehat{Q}(E=0)=\sum_{n \geq 0} Q(n)=0$.

Now, it is clear that if one defines the price process $p_{n}$ as:

$$
p_{n}=\sum_{m<n} \xi_{m}
$$

then $p_{n}$ is a diffusion process with a strictly linear $\mathcal{D}(\ell)$, since the $\xi$ 's are by construction uncorrelated. The price defined in this way can also be written, using Eq. (31), as a linear combination of past $q_{m}$ 's, as assumed in our micromodel Eq. (12), with:

$$
G_{0}^{*}(\ell) \equiv \sum_{m=0}^{\ell-1} Q(m)
$$


This is an exact relation between $\mathcal{C}_{2}$ (that allows one to compute in turn $K$ and $Q)$ and the response function $G_{0}^{*}$ for all $\ell$ 's, where the star indicates that strict diffusion is imposed.

In the case of power-law kernels, one finds from the above relation and from $Q(n) \propto n^{\delta-2}$ for large $n$ :

$$
G_{0}^{*}(\ell) \propto \ell^{\delta-1} \longrightarrow \beta=1-\delta=\frac{1-\gamma}{2},
$$

which is, not surprisingly, the relation obtained in the main text from the assumption that prices are diffusive on long time scales.

Eq. (34) can be used to construct $G_{0}^{*}$ from the empirical determination of $\mathcal{C}_{2}$, shown in Fig. 10. In order to obtain this curve, we have fitted $\mathcal{C}_{2}(n)$ as:

$$
\mathcal{C}_{2}(0)=33.5 ; \quad \mathcal{C}_{2}(n)=\frac{7.16}{(1.95+n)^{0.285}}
$$

and used the Levinson-Durbin recursion algorithm for solving a Toeplitz system (see, e.g., 44]).

\section{References}

[1] E. F. Fama, Efficient capital markets: A review of theory and empirical work, Journal of Finance, 25, 383 (1970).

[2] P. A. Samuelson, Proof that properly anticipated prices fluctuate randomly, Industrial Management Review, 6, 41 (1965).

[3] R. J. Schiller, Do Stock Prices move too much to be justified by subsequent changes in dividends ?, American Economic Review, 71, 421 (1981). R. J. Schiller, Irrational Exuberance, Princeton University Press (2000).

[4] W. B. Arthur, Complexity in Economic and Financial Markets, Complexity, 1, 1 (1995).

[5] A. Shleifer, Inefficient Markets, An Introduction to Behavioral Finance, Oxford University Press (2000).

[6] A. Orléan, Le pouvoir de la finance, Odile Jacob, Paris (1999); A quoi servent les marchés financiers?, in Qu'est-ce que la Culture?, Odile Jacob, Paris (2001).

[7] C. Hopman, Are supply and demand driving stock prices?, MIT working paper, Dec. 2002.

[8] P. Bak, M. Paczuski, and M. Shubik, Price variations in a stock market with many agents, Physica A 246, 430 (1997) 
[9] S. Maslov, Simple model of a limit order-driven market, Physica A 278, 571 (2000); S. Maslov, M. Millis, Price fluctuations from the order book perspective - empirical facts and a simple model, Physica A 299, 234 (2001)

[10] D. Challet, R. Stinchcombe, Analyzing and modelling 1+1d markets, Physica A 300, 285 (2001)

[11] F. Slanina, Mean-field approximation for a limit order driven market model, Phys. Rev. E 64, 056136 (2001).

[12] M. Daniels, J.D. Farmer, G. Iori, E. Smith, Demand storage, market liquidity, and price volatilty, SFI working paper 02-01-001. This paper appeared in final, but truncated form as: M. G. Daniels, J. D. Farmer, G. Iori, E. Smith, Quantitative model of price diffusion and market friction based on trading as a mechanistic random process, Phys. Rev. Lett. 90, 108102 (2003).

[13] J.P. Bouchaud, M. Mézard, M. Potters, Statistical properties of stock order books: empirical results and models, Quantitative Finance 2, 251 (2002).

[14] E. Smith, J. D. Farmer, L. Gillemot, S. Krishnamurthy, Statistical theory of the continuous double auction, e-print cond-mat/0210475, to appear in Quantitative Finance.

[15] D. Challet, R. Stinchcombe, Non-constant rates and over-diffusive prices in a simple model of limit order markets, Quantitative Finance 3, 155 (2003).

[16] M. Wyart, J.P. Bouchaud, in preparation.

[17] J. D. Farmer, talk presented at the ICBI Global Derivatives Trading Summit, Barcelona, May 2003; F. Lillo, J. D. Farmer, in preparation.

[18] C. K. Peng, J. Mietus, J. Hausdorff, S. Havlin, H. E. Stanley, and A. L. Goldberger, Long-Range Anticorrelations and Non-Gaussian Behavior of the Heartbeat, Phys. Rev. Lett. 70, 1343-1346 (1993); P. Bernaola-Galvan, P. Ch. Ivanov, L. A. N. Amaral, and H. E. Stanley, Scale Invariance in the Nonstationarity of Human Heart Rate Phys. Rev. Lett. 87, 168105 (2001). The qualitative analogy with financial markets was recently discussed in: Z. Struzik, Taking the pulse of the economy, Quantitative Finance 3, 78 (2003).

[19] J. L. Cabrera and J. G. Milton, On-Off Intermittency in a Human Balancing Task, Phys. Rev. Lett. 89, 158702 (2002)

[20] T. Lux, The stable Paretian hypothesis and the frequency of large returns: an examination of major German stocks, Applied Financial Economics, 6, 463, (1996). 
[21] V. Plerou, P. Gopikrishnan, L.A. Amaral, M. Meyer, H.E. Stanley, Scaling of the distribution of price fluctuations of individual companies, Phys. Rev. E60 6519 (1999); P. Gopikrishnan, V. Plerou, L. A. Amaral, M. Meyer, H. E. Stanley, Scaling of the distribution of fluctuations of financial market indices, Phys. Rev. E 605305 (1999).

[22] V. Plerou, P. Gopikrishnan, L. A. N. Amaral, X. Gabaix, and H. E. Stanley, Diffusion and Economic Fluctuations, Phys. Rev. E 62, 3023 (2000).

[23] R. Mantegna, H. E. Stanley, An Introduction to Econophysics, Cambridge University Press, Cambridge, 1999; J.-P. Bouchaud and M. Potters, Theory of Financial Risks and Derivative Pricing, Cambridge University Press, Second Edition, 2003.

[24] for a recent review, see: R. Cont, Quantitative Finance, Empirical properties of asset returns: stylized facts and statistical issues, 1, 223 (2001), and refs. therein.

[25] J.-F. Muzy, J. Delour, E. Bacry, Modelling fluctuations of financial time series: from cascade process to stochastic volatility model, Eur. Phys. J. B 17, 537-548 (2000); for a recent review, see: J.P. Bouchaud, J.F. Muzy, Financial time series: from Bachelier's random walk to multifractal 'cascades', to appear in 'Kolmogorov's Legacy', Editions Belin/Springer, Paris/Berlin 2003.

[26] M. Wyart, J.P. Bouchaud, Self-referential behaviour, overreaction and conventions in financial markets, e-print cond-mat/0303584, submitted to J. Economic Behaviour \& Organisation.

[27] F. Black, Noise, J. of Finance, 41529 (1986).

[28] W. de Bondt, R. Thaler, Does the market overreact?, Journal of Finance, XL, 793 (1985).

[29] J. Hasbrouck, Measuring the information content of stock trades, Journal of Finance, XLVI, 179 (1991).

[30] Barra, Market Impact, unpublished report, 1997.

[31] A. Kempf, O. Korn, Market Depth and Order Size, Journal of Financial Markets, 2, 29 (1999).

[32] V. Plerou, P. Gopikrishnan, X. Gabaix, H. E. Stanley, Quantifying Stock Price Response to Demand Fluctuations, Phys. Rev. E 66, 027104 (2002). 
[33] F. Lillo, R. Mantegna, J. D. Farmer, Single Curve Collapse of the Price Impact Function for the New York Stock Exchange, e-print cond-mat/0207428 Master curve for price-impact function, Nature, 421, 129 (2003).

[34] M. Potters, J.P. Bouchaud, More statistical properties of order books and price impact, Physica A, 324, 133 (2003).

[35] T. Odean, Do Investors Trade Too Much?, American Economic Review, 89, 1279 (1999).

[36] B. Rosenow, Fluctuations and Market Friction in Financial Trading, Int. J. Mod. Phys. C, 13, 419 (2002). Somewhat related results, although not discussed in these terms, can also be found in 22 .

[37] J.-P. Bouchaud, R. Cont, A Langevin approach to stock market fluctuations and crashes, European Journal of Physics, B 6, 543 (1998).

[38] on this point, see: X. Gabaix, P. Gopikrishnan, V. Plerou, H. E. Stanley, A theory of power-law distributions in financial markets fluctuations, Nature, 423, 267 (2003). We associate the power-law distribution of investors' size to the long range correlations reported here rather than to the power-law tail of return, as advocated in the above cited paper.

[39] P. Bak, How Nature Works: The Science of Self-Organized Criticality, Copernicus, Springer, New York, 1996.

[40] D. Challet, A. Chessa, M. Marsili, Y.C. Zhang, From Minority Games to real markets, Quantitative Finance, 1, 168 (2001) and refs. therein; D. Challet, M. Marsili, Y.C. Zhang, The Minority Game, book in preparation.

[41] I. Giardina, J. P. Bouchaud, Bubbles, crashes and intermittency in agent based market models, Eur. Phys. Jour. B 31, 421 (2003); see also the discussion in: J.P. Bouchaud, Power-laws in economics and finance: some ideas from physics, Quantitative Finance, 1, 105 (2001).

[42] T. Lux, M. Marchesi, Volatility Clustering in Financial Markets: A Microsimulation of Interacting Agents, Int. J. Theo. Appl. Fin. 3, 675 (2000).

[43] A. Krawiecki, J. A. Hoyst, and D. Helbing, Volatility Clustering and Scaling for Financial Time Series due to Attractor Bubbling, Phys. Rev. Lett. 89, 158701 (2002).

[44] D. B. Percival, Simulating Gaussian Random Processes with Specified Spectra, Computing Science \& Statistics, 24, 534 (1992). 